Supporting Information

\title{
Size-Dependent Hot Carrier Dynamics in Perovskite Nanocrystals Revealed by Two-Dimensional Electronic Spectroscopy
}

Buyang Yu, ${ }^{\dagger, \S}$ Lan Chen, ${ }^{\dagger,} \S$ Zhengkang Qu ${ }^{\dagger, \S}$, Chunfeng Zhang, ${ }^{*}, \dagger$ Zhengyuan Qin, ${ }^{\dagger}$ Xiaoyong Wang, ${ }^{\dagger}$ and Min Xiao, ${ }^{*} \dagger, \ddagger$

${ }^{\dagger}$ National Laboratory of Solid State Microstructures, School of Physics, and

Collaborative Innovation Center for Advanced Microstructures, Nanjing University, Nanjing 210093, China

${ }^{\ddagger}$ Department of Physics, University of Arkansas, Fayetteville, Arkansas 72701, United States 


\section{Synthesis of size-controlled $\mathrm{CsPbI}_{3}$ nanocrystal.}

We synthesized mono-disperse $\mathrm{CsPbI}_{3}$ nanocrystals with varying sizes by modifying methods in recent literatures reported by Luther et al. ${ }^{1}$ and Yao et al. ${ }^{2}$. The precursor is prepared by heating the mixture of $0.625 \mathrm{mmol}$ cesium carbonate $\left(\mathrm{Cs}_{2} \mathrm{CO}_{3}\right), 625 \mu \mathrm{L}$ oleic acid (OA) and $10 \mathrm{~mL}$ octadecene (ODE). Later, $0.375 \mathrm{mmol}$ lead iodide $\left(\mathrm{PbI}_{2}\right)$, $0.225 \mathrm{mmol}$ strontium iodide $\left(\mathrm{SrI}_{2}\right)$ and $10 \mathrm{~mL} \mathrm{ODE}$ are heated at $120^{\circ} \mathrm{C}$ to remove the air. Then add $2.8 \mathrm{~mL}$ of the $1: 1$ mixture of oleylamine $(\mathrm{OAm})$ and $\mathrm{OA}$ preheated to $100^{\circ} \mathrm{C}$ to the solution and keep it under $\mathrm{N}_{2}$ flow for $5 \mathrm{~min}$. The mixture is degassed in vacuum for $15 \mathrm{~min}$ and then heated to intended temperature $\left(130^{\circ} \mathrm{C}, 150^{\circ} \mathrm{C}, 170^{\circ} \mathrm{C}\right.$ and $190^{\circ} \mathrm{C}$, respectively) to control the size of nanocrystals. $0.8 \mathrm{~mL}$ of precursor preheated to $120^{\circ} \mathrm{C}$ was added into the mixture quickly. Then the whole mixture was put into the ice-water mixture to cool down to room temperature. After that, ethyl acetate is added with a ratio of 4:1 before centrifuging at $10000 \mathrm{rpm}$ for $5 \mathrm{~min}$. The deposition resolved into hexane is centrifuged again at $10000 \mathrm{rpm}$ for $10 \mathrm{~min}$. For samples reacting at $130^{\circ} \mathrm{C}$, an extra cooling under icy water for $3 \mathrm{~h}$ is necessary before centrifugation. The average sizes of the samples are about 4.6, 6.1, 6.7 and $8.2 \mathrm{~nm}$.

\section{Optical characterizations}

Our setup for two-dimensional electronic spectroscopy (2DES) is based on pumpprobe geometry, combining with active phase stabilization. ${ }^{3}$ Two home-made noncollinear optical parametric amplifiers (NOPAs) were exploited as pump and probe sources. ${ }^{4}$ The beam $(800 \mathrm{~nm}, 90 \mathrm{fs}, 1 \mathrm{kHz})$ of a Ti:sapphire regenerative amplifier 
(Libra, Coherent) was employed to pump NOPAs. The pump and probe beams were compressed by chirper mirrors and optical wedges to achieve the ultrashort pulse duration of $8.8 \mathrm{fs}$, which can be measured by fringe-resolved autocorrelation (FRAC, Figure S1). For all samples, the centeral energies of the pump spectra were tuned to $0.15 \mathrm{eV}$ higher than the excitonic transition energies. Two phase-locked pump beams with time delay $\tau$ were split from the first NOPA laser and re-combined to excite the sample. The probe beam was delayed by a population time $\mathrm{T}$ after the second pump laser. In our configuration, a fiber spectrometer (Maya 2000 Pro, Ocean Optics) was used to monitor the phase difference between the two pump beams. The transmitted probe was collected by a monochromator (HRS-750, Princeton Instruments) and analyzed by a charge-coupled device (CCD, S11071, Hamamatsu) at $1 \mathrm{KHz}$. For heterodyne detection, the pump beams were modulated by a chopper, while the probe beam (also as local oscillator) was detected by the spectrometer to calculate $\Delta \mathrm{T} / \mathrm{T}_{0}{ }^{0.5}$. The $\mathrm{T}_{0}$ denominator corresponds to the spectra due to probe laser only when pump beams are off. The $\Delta \mathrm{T}$ in numerator relates to the difference of the probe spectra while pump beams are on versus off. The average excitation density per nanocrystal is determined by the pump spectrum and the calculated aborption cross section involved in the pump spectrum.

\section{Analysis of hot carrier distribution}

The calculated TA signal is generated by integrating the 2DES signal along the excitation axis and then dividing by the corresponding $\mathrm{T}_{0}{ }^{0.5}$. The hot-carrier temperature is extracted by fitting the high-energy tail in the calculated TA. After thermalization process, the quasi-equilibrium carrier distribution can be characterized by a Fermi- 
Dirac distribution function. Due to the large energy difference between the energy of carrier and the Fermi level (compared to $\mathrm{k}_{\mathrm{B}} \mathrm{T} \sim 25 \mathrm{meV}$ at room temperature), the Fermi-Dirac distribution function can be approximated as a Maxwell-Boltzmann distribution function.

$$
f \sim \exp \frac{E_{F}-E}{k_{B} T_{C}}
$$

$\mathrm{k}_{\mathrm{B}}$ is the Boltzmann constant and $\mathrm{E}_{\mathrm{f}}$ is the quasi-Fermi energy. We choose a high energy region with $0.2 \mathrm{eV}$ width. ${ }^{5}$ The evolution of carrier temperature $\mathrm{T}_{\mathrm{C}}$ can reflect the variation of hot carrier distribution and thus the process of hot carrier relaxation as extensively used in previous studies. ${ }^{6-8}$

\section{Energy loss rate}

The hot carrier energy loss rate was determined by $\Delta \mathrm{E} / 2 \mathrm{t}_{\mathrm{r}} . \Delta \mathrm{E}$ is the energy difference between the initial hot state to the cooled state that is excess energy. $t_{r}$ is the cooling lifetime parameter. The factor 2 is determined due to the comparable effective masses of electron and hole in $\mathrm{CsPbI}_{3}$ nanocrystals. ${ }^{9}$ 


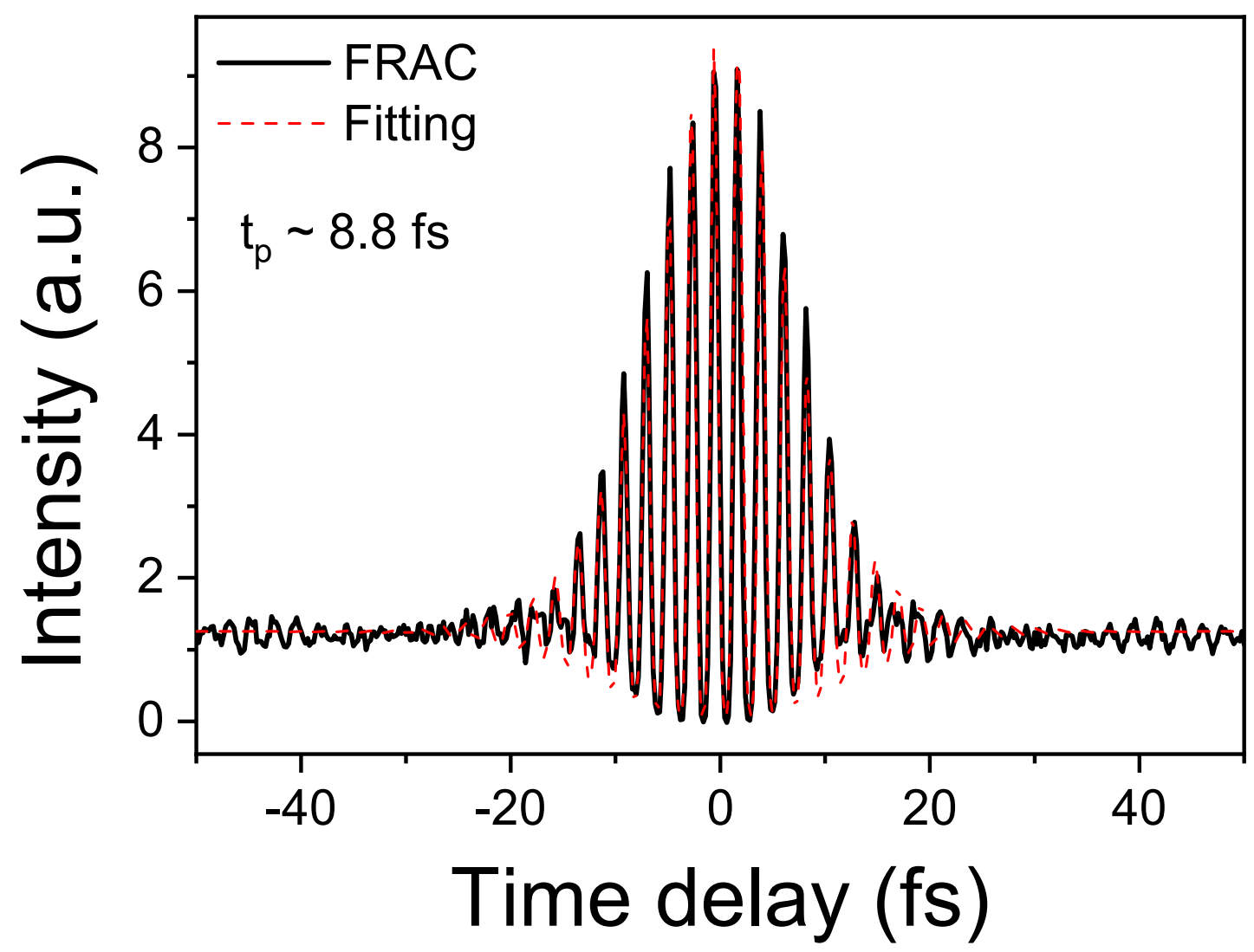

Figure S1. FRAC trace of the pump pulse (black solid line) and a $\mathrm{sech}^{2}$ - fit curve (red dashed line) show the pulse width is $\sim 8.8 \mathrm{fs}$. 

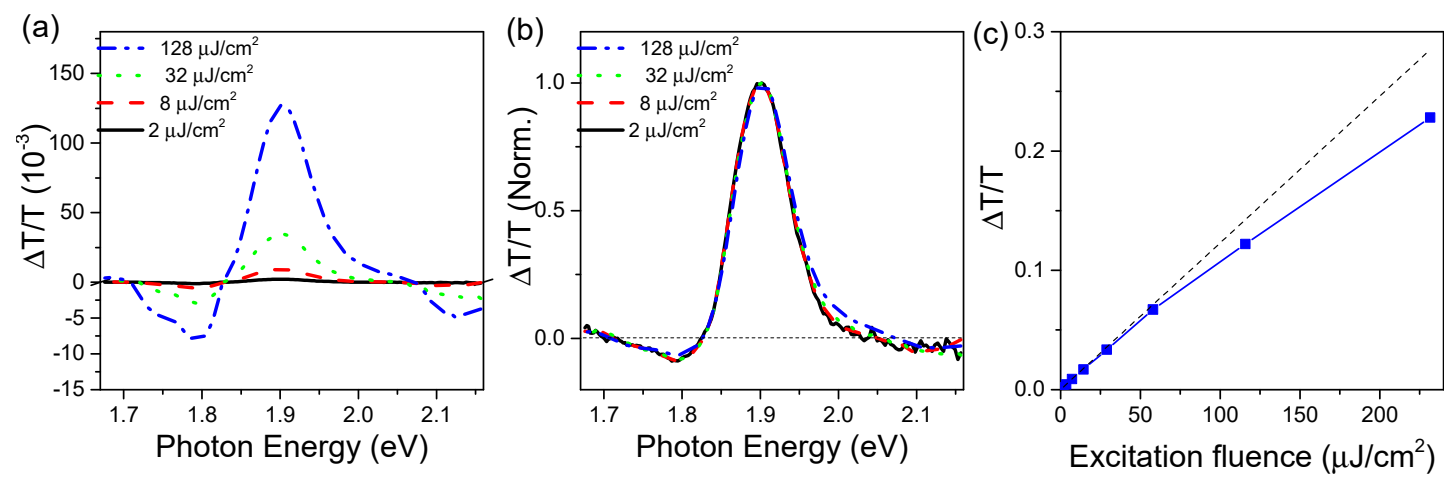

Figure S2. Power-dependent transient absorption (TA) spectroscopic measurements. (a) TA spectra of a solution sample of $\mathrm{CsPbI}_{3}$ nanocrystals recorded at the delay of $100 \mathrm{fs}$ upon pump of different fluences. (b) Normalized TA spectra in panel (a). (c) The amplitude of bleach signal is plotted versus excitation fluence. 

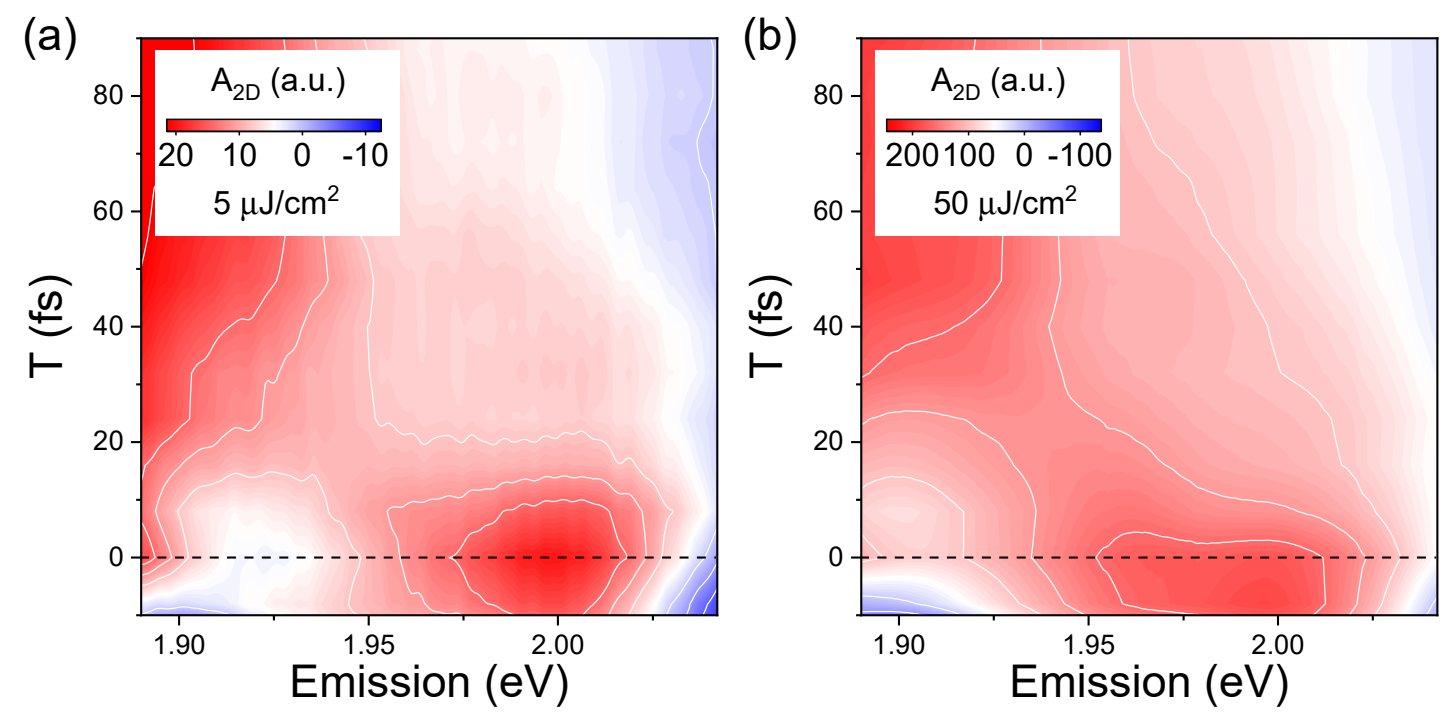

Figure S3. Counter plot of emission spectra of different excitation fluence (a) $5 \mu \mathrm{J} \mathrm{cm}$ ${ }^{2}$ and (b) $50 \mu \mathrm{J} \mathrm{cm}^{-2}$ at early-stage ( $<100 \mathrm{fs}$ ) extracted from 2DES data. Under higher excitation fluence, the broader bleaching signal around the excitation energy reflect the enhanced carrier-carrier scattering. The data were recorded from the nanocrystal sample with the average size of $6.7 \mathrm{~nm}$. 

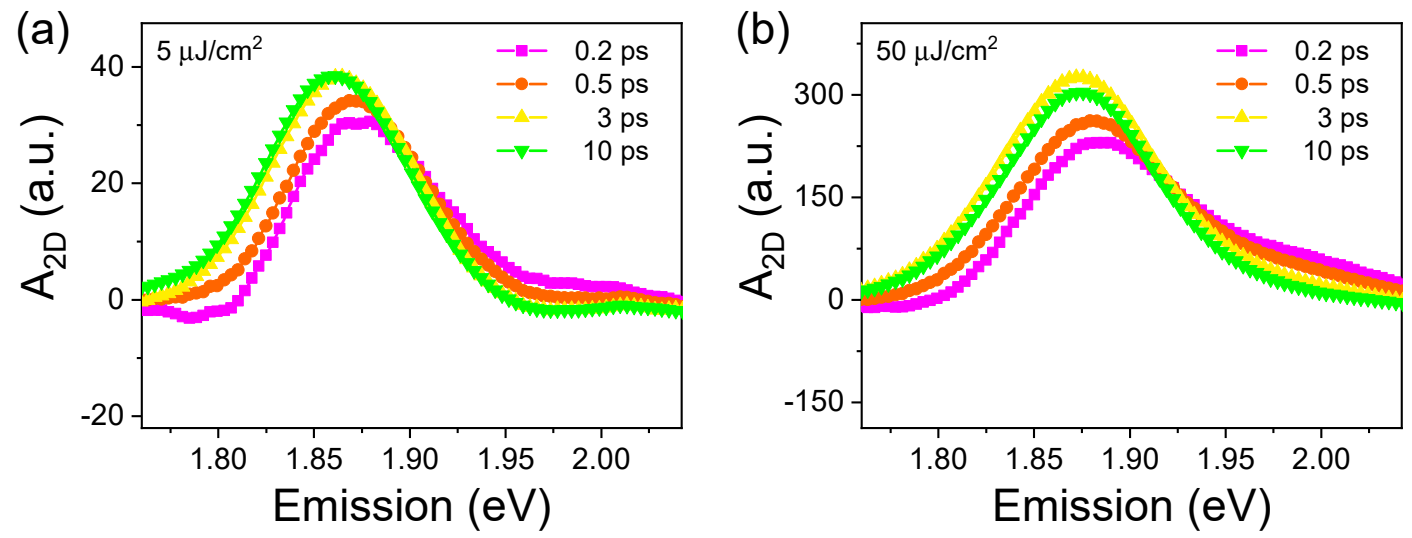

Figure S4. Emission spectra of different excitation fluence (a) $5 \mu \mathrm{J} \mathrm{cm}^{-2}$ and (b) $50 \mu \mathrm{J}$ $\mathrm{cm}^{-2}$ extracted from 2DES data at different population delays (T). The spectra under high excitation fluence show a broader energy distribution. The data were recorded from the nanocrystal sample with the average size of $6.7 \mathrm{~nm}$ with the excitation excess energy $\Delta \mathrm{E}=0.15 \mathrm{eV}$. 

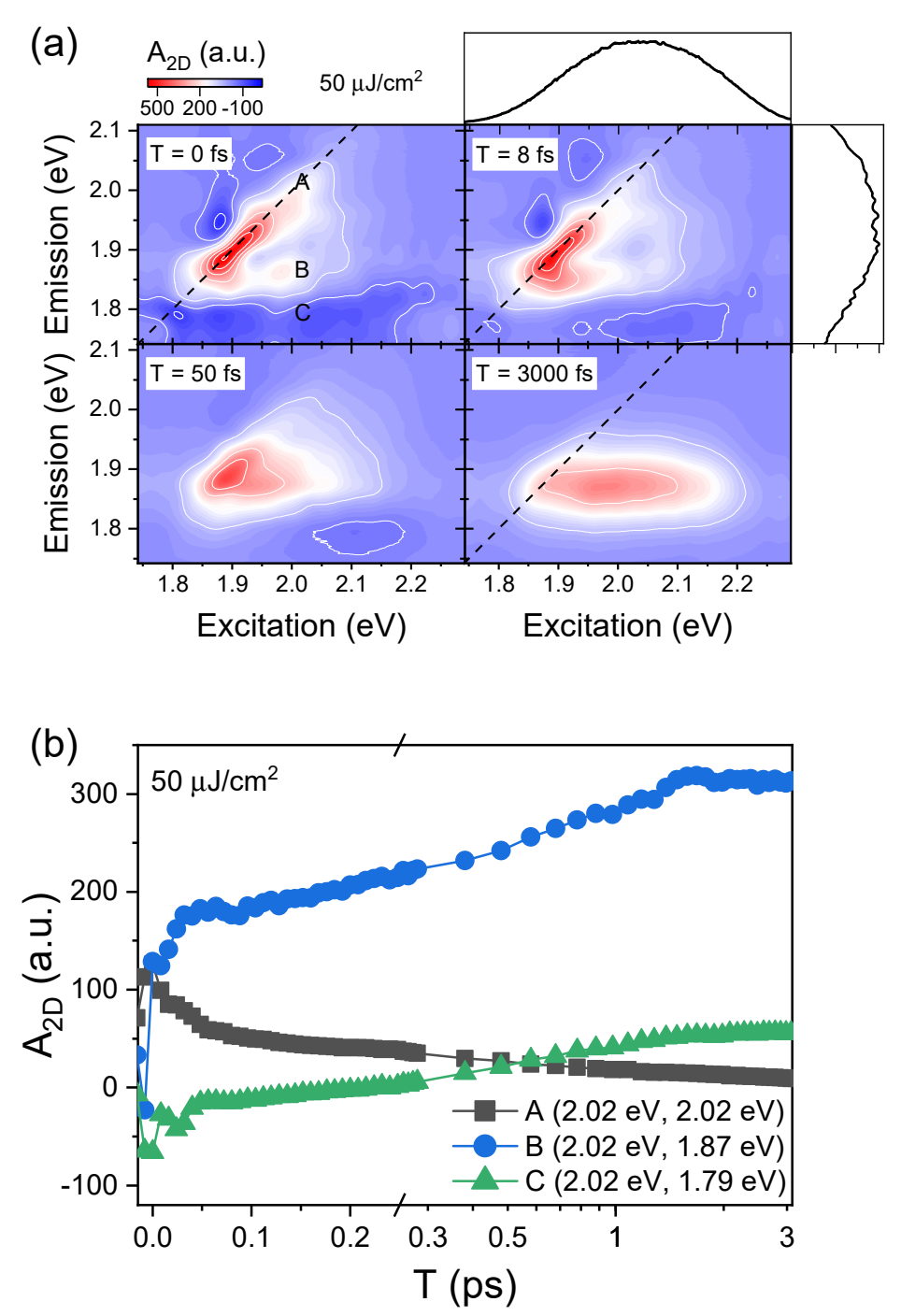

Figure S5. 2DES data recorded under high excitation fluence $\left(50 \mu \mathrm{J} \mathrm{cm}^{-2}\right)$. (a) Absorptive 2DES spectra recorded at representative population delays of $\mathrm{T}=0,8,50$, 3000 fs. The diagonal is indicated with the dashed lines. (b) Temporal dynamics of 2DES signal probed at excitation excess energy $\Delta \mathrm{E}=0.15 \mathrm{eV}$ and different emission energies marked as A, B and C in panel (a). The data were recorded from the sample with average size of $6.7 \mathrm{~nm}$. 

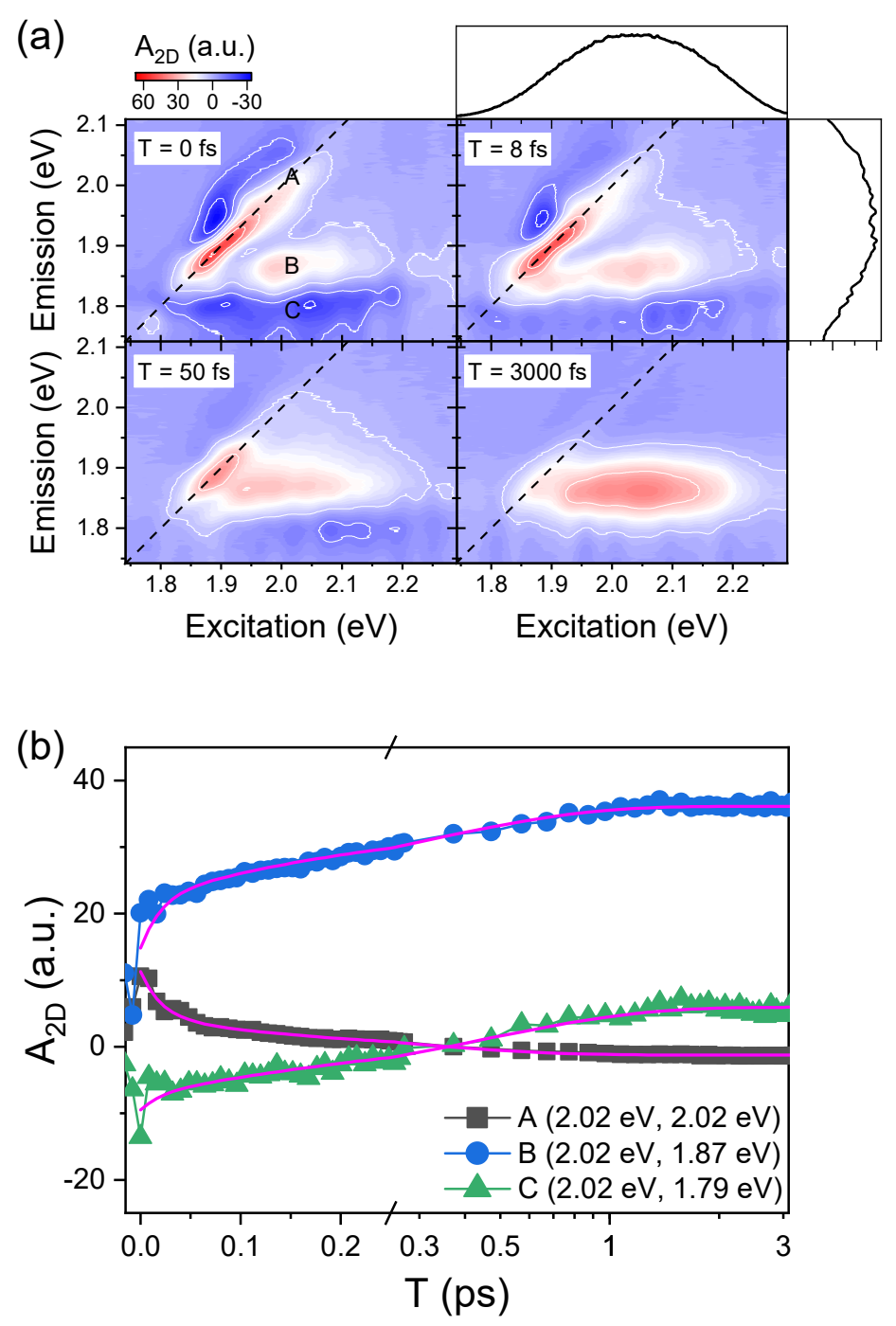

Figure S6. (a) Absorptive 2DES spectra recorded at representative population delays of $\mathrm{T}=0,8,50,3000 \mathrm{fs}$. The diagonal is indicated with the dashed lines. (b) Temporal dynamics of 2DES signal probed at excitation excess energy of $\Delta \mathrm{E}=0.15 \mathrm{eV}$ and different emission energies marked as A, B and C in panel (a). The curves exhibit twostage dynamics which can be reproduced by a biexponential function with lifetime parameters of $\sim 20 \mathrm{fs}$ and $\sim 320 \mathrm{fs}$ (pink lines), respectively. The data were recorded from the sample with an average size of $6.7 \mathrm{~nm}$ and an excitation fluence of $5 \mu \mathrm{J} \mathrm{cm}^{-2}$. 

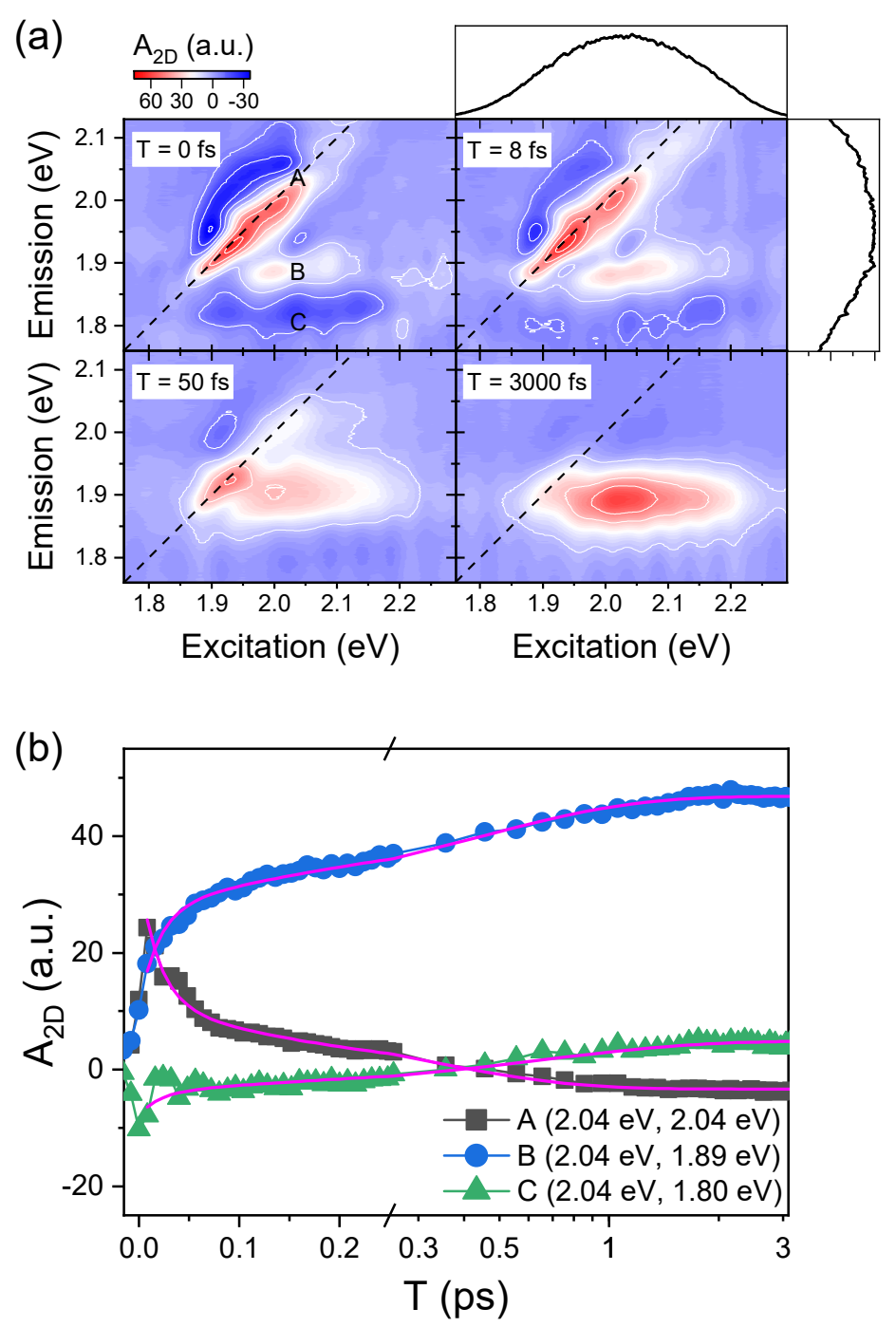

Figure S7. (a) Absorptive 2DES spectra recorded at representative population delays of $\mathrm{T}=0,8,50,3000 \mathrm{fs}$. The diagonal is indicated with the dashed lines. (b) Temporal dynamics of 2DES signal probed at excitation excess energy of $\Delta \mathrm{E}=0.15 \mathrm{eV}$ and different emission energies marked as A, B and C in panel (a). The curves exhibit twostage dynamics which can be reproduced by a biexponential function with lifetime parameters of $\sim 20$ fs and $\sim 430$ fs (pink lines), respectively. The data were recorded from the sample with an average size of $6.1 \mathrm{~nm}$ and an excitation fluence of $5 \mu \mathrm{J} \mathrm{cm}^{-2}$. 

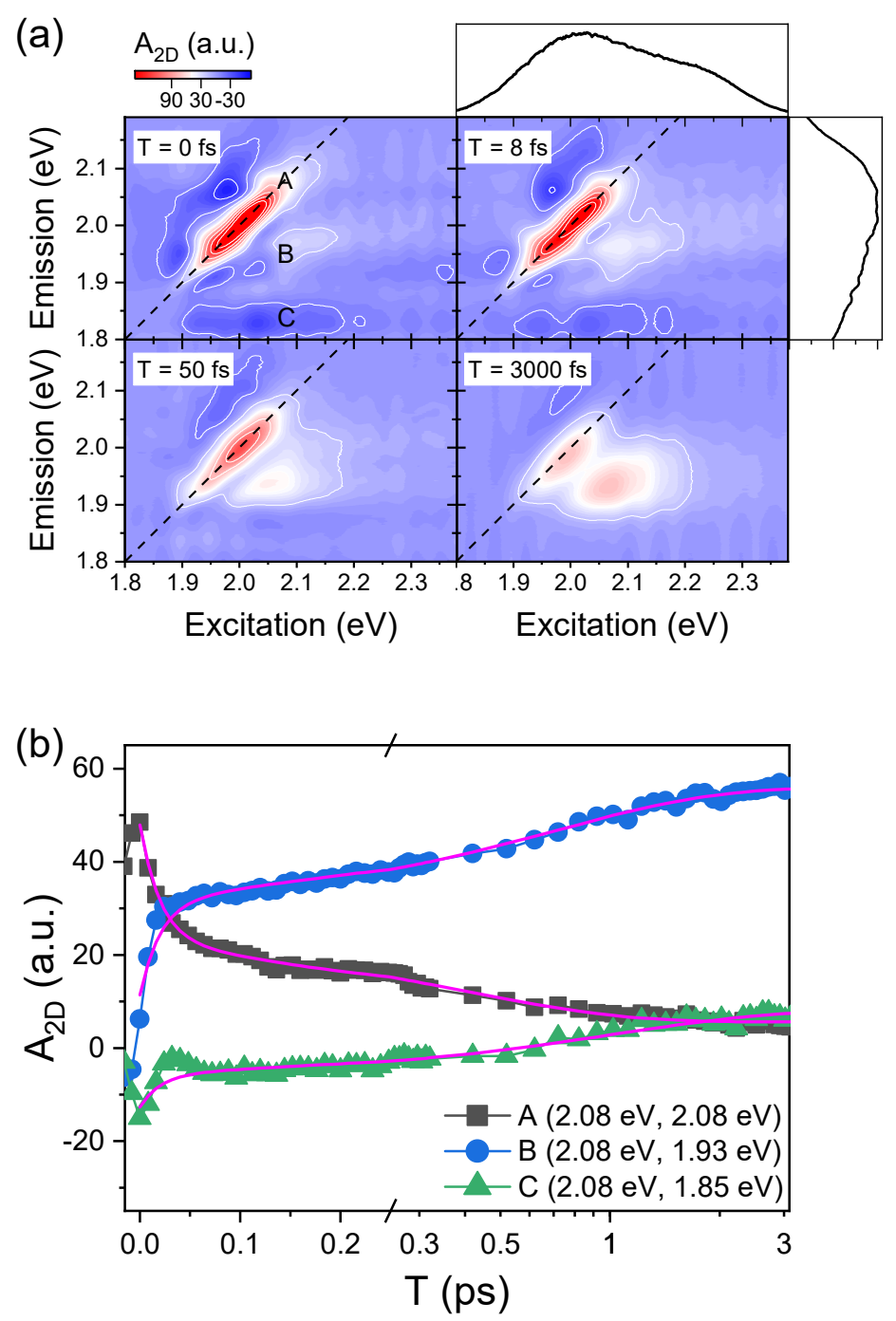

Figure S8. (a) Absorptive 2DES spectra recorded at representative population delays of $\mathrm{T}=0,8,50,3000 \mathrm{fs}$. The diagonal is indicated with the dashed lines. (b) Temporal dynamics of 2DES signal probed at excitation excess energy of $\Delta \mathrm{E}=0.15 \mathrm{eV}$ and different emission energies marked as A, B and C in panel (a). The curves exhibit twostage dynamics which can be reproduced by a biexponential function with lifetime parameters of $\sim 20 \mathrm{fs}$ and $\sim 700 \mathrm{fs}$ (pink lines), respectively. The data were recorded from the sample with an average size of $4.6 \mathrm{~nm}$ and an excitation fluence of $5 \mu \mathrm{J} \mathrm{cm}^{-2}$. 

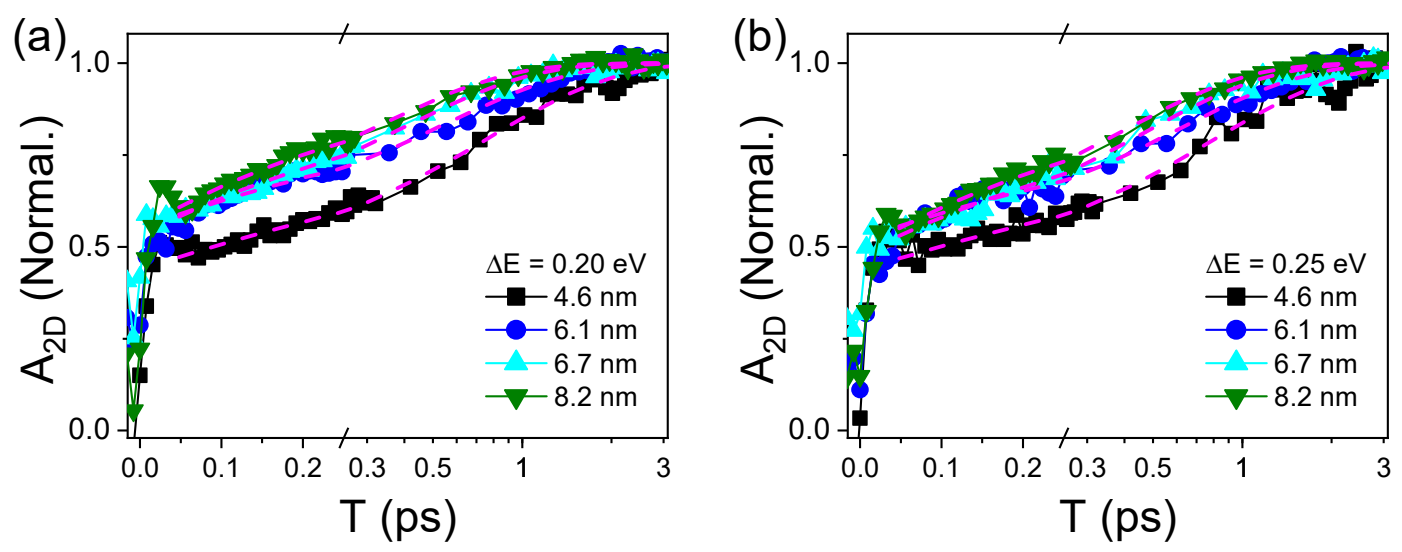

Figure S9. Normalized time-resolved traces of 2D signal probed at the band edge from $\mathrm{CsPbI}_{3}$ nanocrystals of different sizes with excess energy (a) $\Delta \mathrm{E}=0.20 \mathrm{eV}$, (b) $\Delta \mathrm{E}=$ $0.25 \mathrm{eV}$. The excitation fluence is set at $5 \mu \mathrm{J} \mathrm{cm}^{-2}$. The dashed line shows the exponential fitting of hot carrier cooling. 


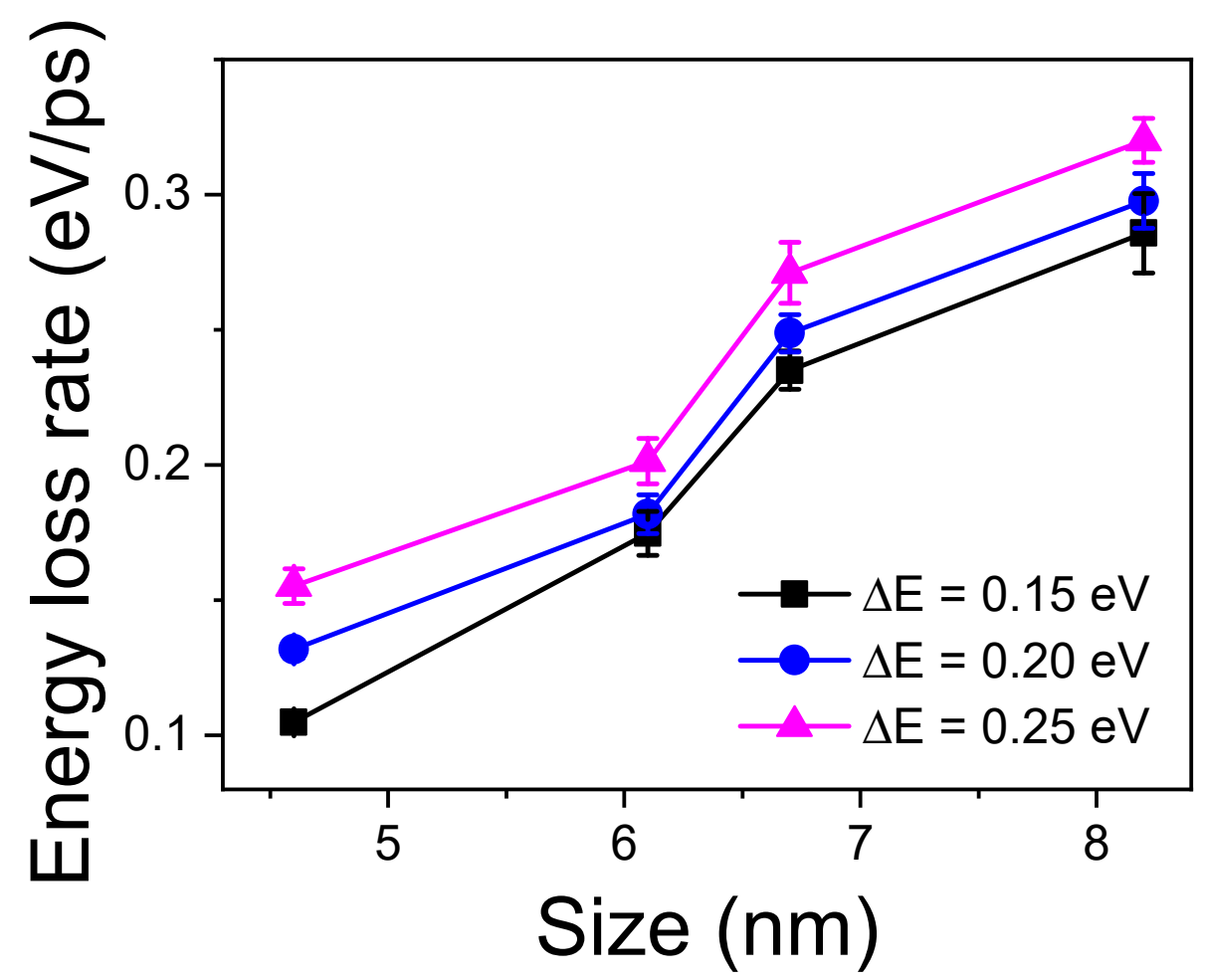

Figure S10. Size-dependent energy loss rate for hot carrier dynamics. The energy loss rate slows down with reducing nanocrystal size as a signature of phonon bottleneck effect. 


\section{References}

(1) Swarnkar, A.; Marshall, A. R.; Sanehira, E. M.; Chernomordik, B. D.; Moore, D. T.; Christians, J. A.; Chakrabarti, T.; Luther, J. M. Quantum dot-induced phase stabilization of $\alpha-\mathrm{CsPI}_{3}$ perovskite for high-efficiency photovoltaics. Science 2016, 354 (6308), 92-95.

(2) Yao, J. S.; Ge, J.; Wang, K. H.; Zhang, G.; Zhu, B. S.; Chen, C.; Zhang, Q.; Luo, Y.; Yu, S. H.; Yao, H. B. Few-nanometer-sized alpha-CsPbI ${ }_{3}$ quantum dots enabled by strontium substitution and iodide passivation for efficient red-light emitting diodes. J. Am. Chem. Soc. 2019, 141 (5), 2069-2079.

(3) Zhu, W.; Wang, R.; Zhang, C.; Wang, G.; Liu, Y.; Zhao, W.; Dai, X.; Wang, X.; Cerullo, G.; Cundiff, S.; Xiao, M. Broadband two-dimensional electronic spectroscopy in an actively phase stabilized pump-probe configuration. Opt. Express 2017, 25 (18), 21115.

(4) Cerullo, G.; Nisoli, M.; Stagira, S.; De Silvestri, S. Sub-8-fs pulses from an ultrabroadband optical parametric amplifier in the visible. Opt. Lett. 1998, 23 (16), 1283-1285.

(5) Lim, J. W. M.; Giovanni, D.; Righetto, M.; Feng, M.; Mhaisalkar, S. G.; Mathews, N.; Sum, T. C. Hot carriers in halide perovskites: How hot truly? J. Phys. Chem. Lett. 2020, 11 (7), 2743-2750.

(6) Fu, J.; Xu, Q.; Han, G.; Wu, B.; Huan, C. H. A.; Leek, M. L.; Sum, T. C. Hot carrier cooling mechanisms in halide perovskites. Nat. Commun. 2017, 8 (1), 1300.

(7) Price, M. B.; Butkus, J.; Jellicoe, T. C.; Sadhanala, A.; Briane, A.; Halpert, J. E.; Broch, K.; Hodgkiss, J. M.; Friend, R. H.; Deschler, F. Hot-carrier cooling and photoinduced refractive index changes in organic-inorganic lead halide perovskites. Nat. Commun. 2015, 6 (1), 8420.

(8) Yang, J.; Wen, X.; Xia, H.; Sheng, R.; Ma, Q.; Kim, J.; Tapping, P.; Harada, T.; Kee, T. W.; Huang, F.; Cheng, Y. B.; Green, M.; Ho-Baillie, A.; Huang, S.; Shrestha, S.; Patterson, R.; Conibeer, G. Acoustic-optical phonon up-conversion and hot-phonon bottleneck in lead-halide perovskites. Nat. Commun. 2017, 8 (1), 14120.

(9) Protesescu, L.; Yakunin, S.; Bodnarchuk, M. I.; Krieg, F.; Caputo, R.; Hendon, C. H.; Yang, R. X.; Walsh, A.; Kovalenko, M. V. Nanocrystals of cesium lead halide perovskites $\left(\mathrm{CsPbX}_{3}, \mathrm{X}=\mathrm{Cl}, \mathrm{Br}\right.$, and I): Novel optoelectronic materials showing bright emission with wide color gamut. Nano Lett. 2015, 15 (6), 3692-3696. 